Proceedings of the 2005 Winter Simulation Conference

M. E. Kuhl, N. M. Steiger, F. B. Armstrong, and J. A. Joines, eds.

\title{
SIMULATION VALIDATION WITH HISTORIC OUTCOMES
}

\author{
Lance E. Champagne \\ Air Force Logistics Management Agency \\ 501 Ward Street \\ Maxwell AFB, Gunter Annex, AL 36114-3236, U.S.A.
}

\author{
Raymond R. Hill \\ Wright State University \\ Dept. of Biomed., Ind., and Human Fact. Engr. \\ 207 Russ Engineering Center \\ 3640 Colonel Glenn Hwy. \\ Dayton, OH 45435-0001. U.S.A.
}

\begin{abstract}
Combat, unlike many real-world processes, tends to be singular in nature. That is, there are not multiple occurrences from which to hypothesize a probability distribution model of the real-world system. Mission-level models may offer more flexibility on some measures due to their extended time frame. Additionally, the parameters involved in the mission-level model may be unchanged for significant stretches of the total simulation time. In these cases, time periods may be devised so that the periods hold sufficiently similar traits such that the incremental results may be assumed to come from a common distribution. This paper details a new statistical methodology for use in validating an agent-based mission-level model. The test is developed within the context of the Bay of Biscay agentbased simulation and uses the monthly data from the extended campaign as a basis of comparison to the simulation output.
\end{abstract}

\section{INTRODUCTION}

In recent years, there has been an increased number of agent-based simulations studying various aspects of combat. For example, Tighe, in (Tighe 1999), developed an agent-based simulation based ultimately on the boids flocking algorithm (Levy 1992) and ISAAC (Ilachinski 1998) as an attempt to find a method of quantifying strategic effects, purported to be one of the main strengths of air power in combat. Bullock, in (Bullock 2000), continued the research into modeling strategic effects with the introduction of the Hierarchical Interactive Theater Model (HITM). This model was intended to provide a sufficiently complex tool able to show strategic effects of air power, while retaining enough simplicity to allow identification of interactions between important factors. Other agent-based combat simulation research includes modeling riot tactics for small military units (Woodaman 2000), small unit peacekeeping tactics in an urban environment (Brown
2000), and a German training scenario involving small units over a relatively short time period (Erlenbruch 2002).

Though each of the above provides significant results toward advancing the field of agent-based combat simulation, no attempt was made to relate simulation outcome to real-world data. This paper outlines the development of a statistical validation technique applied to an agent-based combat simulation based on the Allied offensive against the German U-Boats in the Bay of Biscay during WW II. Model results are compared to the historical data.

\subsection{Historical Scenario}

German U-Boats operated against Allied shipping in the North Atlantic from 1941 through the end of the war in an effort to reduce the shipments of war-time supplies to Great Britain. Following the fall of France, many of these submarines operated from ports in occupied France, crossing the Bay of Biscay into the North Atlantic, where they hunted for Allied transport ships. Once they left the Bay of Biscay, the U-Boats could, for all practical purposes, operate outside the reach of Allied aircraft support. For a time in 1942 and 1943, this offensive was so successful, that Great Britain's war effort was put in great peril.

While the Allied forces had little hope of finding and destroying U-Boats once they reached the Atlantic, the Bay of Biscay was well within the reach of Allied aircraft. Additionally, the amount of U-Boat traffic to and from the French ports, necessitated by maintenance and resupply/refuel demands, ultimately meant that there was sufficient density of targets within the Bay of Biscay to warrant committing resources to conduct anti-U-Boat efforts there. As a result, the Allied forces, beginning in 1941, hunted for the U-Boats in the Bay of Biscay.

Additional historical background on the offensive search in the Bay of Biscay can be found in (McCue 1990), and an extensive record of the corresponding operational analysis may be found in (Waddington 1973) and (Morse and Kimball 1998). 


\section{Champagne and Hill}

\subsection{Bay Of Biscay Model}

Though the Bay of Biscay simulation was built to reproduce the results of the historical operation in both qualitative and quantitative measures, one of the development goals was to keep the simulation relatively simple by including only the most significant factors. As a result, it was necessary to make assumptions about the environment, the aircraft agents, and the U-Boat agents. Detailed descriptions of the Bay of Biscay modeling assumptions may be found in (Champagne 2003a, Champagne 2003b, Champagne and Hill 2003).

\subsection{Simulation Scenarios}

Two scenarios were chosen for the initial model validation. The first was the six month period from October 1942 March 1943 (Scenario 1), and the second was April 1943 September 1943 (Scenario 2). These time periods were chosen because the technologies used by both Allied aircraft and German U-Boats remained relatively constant over the months within each scenario, although they did vary significantly between scenarios.

The U-Boat fleet initially consists of 70 agents distributed randomly and uniformly throughout the Bay of Biscay. A simulation warm up period of 12 months is used to position the fleet through normal movement through the bay and time spent in operational zones and ports. This yields a more natural U-Boat fleet configuration as might have been the real-world case. U-Boat fleet reinforcements begin arriving from Germany according to their historical numbers (McCue 1990) in month 11 of the warm up period and continue throughout the remainder of the simulation.

The aircraft fleet consists of 15 aircraft agents in Scenario 1 and 35 aircraft agents in Scenario 2, collocated at a single airbase in Great Britain. These aircraft numbers were derived through experimentation on the two scenarios until the average monthly flying hours compared favorably with the historical values for flying hours. The number of aircraft agents remains constant throughout each scenario .

Aircraft offensive search is assigned to a fixed area of the bay $200 \times 350 \mathrm{NM}^{2}(\mathrm{E}-\mathrm{W} \times \mathrm{N}-\mathrm{S})$. The search area is subdivided into $50 \times 50 \mathrm{NM}^{2}$ non-overlapping search grids. Aircraft search each grid using a modified barrier search pattern constructed from the tactics discussed in (Waddington 1973).

\section{SIMULATION RESULTS}

Each simulation scenario was replicated 20 times, and statistics were kept for the 6-month total and on a per-month basis. The historical values for each scenario are found in Tables 1 and 2, respectively. The simulation results for Scenario 1 MOEs are found in Tables 3 and 4, respectively, for all 20 replications.
Table 1: Historical MOE Values for Scenario 1 (McCue, 1990)

\begin{tabular}{|l|c|c|c|c|c|c|}
\hline MOE & $10 / 42$ & $11 / 42$ & $12 / 42$ & $1 / 43$ & $2 / 43$ & $3 / 43$ \\
\hline Sightings & 18 & 19 & 14 & 10 & 32 & 42 \\
\hline Kills & 1 & 1 & 0 & 0 & 0 & 1 \\
\hline
\end{tabular}

Table 2: Historical MOE Values for Scenario 2 (McCue, 1990)

\begin{tabular}{|l|c|c|c|c|c|c|}
\hline MOE & $4 / 43$ & $5 / 43$ & $6 / 43$ & $7 / 43$ & $8 / 43$ & $9 / 43$ \\
\hline Sightings & 52 & 98 & 60 & 81 & 7 & 21 \\
\hline Kills & 1 & 7 & 4 & 13 & 5 & 2 \\
\hline
\end{tabular}

Table 3: Simulated U-Boat Sightings for Scenario 1

\begin{tabular}{|c|c|c|c|c|c|c|}
\hline Rep. & $10 / 42$ & $11 / 42$ & $12 / 42$ & $1 / 43$ & $2 / 43$ & $3 / 43$ \\
\hline 1 & 9 & 17 & 21 & 17 & 11 & 33 \\
\hline 2 & 19 & 14 & 25 & 24 & 24 & 23 \\
\hline 3 & 16 & 23 & 15 & 22 & 25 & 28 \\
\hline 4 & 20 & 17 & 21 & 33 & 26 & 33 \\
\hline 5 & 15 & 16 & 18 & 25 & 28 & 26 \\
\hline 6 & 18 & 21 & 20 & 29 & 23 & 32 \\
\hline 7 & 11 & 20 & 24 & 30 & 34 & 28 \\
\hline 8 & 20 & 17 & 17 & 25 & 28 & 23 \\
\hline 9 & 27 & 25 & 34 & 40 & 28 & 30 \\
\hline 10 & 17 & 17 & 26 & 30 & 33 & 45 \\
\hline 11 & 9 & 9 & 23 & 13 & 21 & 27 \\
\hline 12 & 15 & 17 & 27 & 34 & 27 & 39 \\
\hline 13 & 12 & 14 & 18 & 21 & 17 & 25 \\
\hline 14 & 12 & 15 & 15 & 26 & 21 & 27 \\
\hline 15 & 13 & 17 & 16 & 24 & 25 & 36 \\
\hline 16 & 22 & 14 & 16 & 16 & 27 & 25 \\
\hline 17 & 21 & 15 & 23 & 17 & 21 & 23 \\
\hline 18 & 22 & 21 & 22 & 21 & 27 & 36 \\
\hline 19 & 21 & 28 & 32 & 30 & 24 & 21 \\
\hline 20 & 13 & 15 & 22 & 27 & 27 & 26 \\
\hline
\end{tabular}

\subsection{Analysis Of The Simulations MOEs}

Joint confidence intervals around the simulation means can be constructed using a t-statistic, as shown in (3).

$$
\text { Bound }=\bar{x} \pm \frac{s}{\sqrt{n}} \cdot t_{\frac{\alpha}{2 \cdot k}, n-1}
$$

where

- $\bar{x}$ is the sample mean

- $\quad s$ is the sample standard deviation

- $n$ is the sample size

- $\quad k$ is the number of joint confidence intervals

- $(1-\alpha)$ is the desired level of joint confidence. 
Table 4: Simulated U-Boat Kills for Scenario 1

\begin{tabular}{|c|c|c|c|c|c|c|}
\hline Rep. & $10 / 42$ & $11 / 42$ & $12 / 42$ & $1 / 43$ & $2 / 43$ & $3 / 43$ \\
\hline 1 & 0 & 0 & 0 & 1 & 0 & 1 \\
\hline 2 & 0 & 0 & 1 & 1 & 2 & 1 \\
\hline 3 & 0 & 0 & 1 & 1 & 0 & 1 \\
\hline 4 & 0 & 0 & 1 & 0 & 1 & 1 \\
\hline 5 & 0 & 0 & 1 & 1 & 2 & 0 \\
\hline 6 & 0 & 0 & 0 & 1 & 1 & 0 \\
\hline 7 & 0 & 0 & 1 & 2 & 1 & 1 \\
\hline 8 & 0 & 1 & 0 & 0 & 1 & 1 \\
\hline 9 & 1 & 0 & 2 & 1 & 1 & 0 \\
\hline 10 & 1 & 1 & 2 & 1 & 1 & 0 \\
\hline 11 & 1 & 1 & 0 & 1 & 1 & 0 \\
\hline 12 & 1 & 0 & 1 & 0 & 1 & 0 \\
\hline 13 & 1 & 0 & 1 & 0 & 0 & 0 \\
\hline 14 & 0 & 0 & 0 & 0 & 1 & 1 \\
\hline 15 & 0 & 0 & 1 & 1 & 1 & 1 \\
\hline 16 & 2 & 1 & 0 & 0 & 1 & 0 \\
\hline 17 & 0 & 0 & 1 & 1 & 1 & 0 \\
\hline 18 & 0 & 0 & 2 & 1 & 0 & 2 \\
\hline 19 & 0 & 1 & 1 & 2 & 0 & 1 \\
\hline 20 & 0 & 1 & 1 & 0 & 1 & 1 \\
\hline
\end{tabular}

Table 5: Simulated U-Boat Sightings for Scenario 2

\begin{tabular}{|c|c|c|c|c|c|c|}
\hline Rep. & $4 / 43$ & $5 / 43$ & $6 / 43$ & $7 / 43$ & $8 / 43$ & $9 / 43$ \\
\hline 1 & 38 & 50 & 44 & 46 & 45 & 64 \\
\hline 2 & 48 & 46 & 49 & 57 & 62 & 70 \\
\hline 3 & 46 & 43 & 46 & 43 & 57 & 69 \\
\hline 4 & 46 & 48 & 51 & 56 & 69 & 48 \\
\hline 5 & 40 & 49 & 48 & 69 & 70 & 69 \\
\hline 6 & 60 & 46 & 67 & 70 & 58 & 57 \\
\hline 7 & 50 & 46 & 66 & 57 & 59 & 63 \\
\hline 8 & 42 & 52 & 46 & 54 & 74 & 79 \\
\hline 9 & 43 & 60 & 47 & 62 & 70 & 75 \\
\hline 10 & 46 & 53 & 54 & 72 & 75 & 73 \\
\hline 11 & 40 & 44 & 49 & 68 & 56 & 55 \\
\hline 12 & 36 & 59 & 51 & 67 & 63 & 58 \\
\hline 13 & 44 & 29 & 47 & 52 & 55 & 55 \\
\hline 14 & 35 & 40 & 49 & 45 & 71 & 48 \\
\hline 15 & 44 & 44 & 57 & 73 & 58 & 58 \\
\hline 16 & 42 & 58 & 54 & 61 & 60 & 68 \\
\hline 17 & 42 & 47 & 62 & 69 & 71 & 66 \\
\hline 18 & 43 & 59 & 56 & 79 & 74 & 65 \\
\hline 19 & 48 & 53 & 47 & 64 & 72 & 60 \\
\hline 20 & 41 & 45 & 57 & 61 & 59 & 75 \\
\hline
\end{tabular}

Table 6: Simulated U-Boat Kills for Scenario 2

\begin{tabular}{|c|c|c|c|c|c|c|}
\hline Rep. & $4 / 43$ & $5 / 43$ & $6 / 43$ & $7 / 43$ & $8 / 43$ & $9 / 43$ \\
\hline 1 & 0 & 6 & 7 & 3 & 6 & 6 \\
\hline 2 & 1 & 3 & 4 & 8 & 5 & 5 \\
\hline 3 & 6 & 5 & 5 & 5 & 4 & 3 \\
\hline 4 & 2 & 9 & 4 & 3 & 9 & 3 \\
\hline 5 & 2 & 2 & 5 & 4 & 6 & 9 \\
\hline 6 & 4 & 5 & 8 & 8 & 8 & 5 \\
\hline 7 & 6 & 2 & 12 & 9 & 4 & 6 \\
\hline 8 & 3 & 2 & 8 & 8 & 9 & 13 \\
\hline 9 & 4 & 5 & 1 & 5 & 6 & 7 \\
\hline 10 & 5 & 4 & 4 & 6 & 13 & 5 \\
\hline 11 & 7 & 7 & 3 & 9 & 6 & 2 \\
\hline 12 & 6 & 3 & 2 & 12 & 9 & 5 \\
\hline 13 & 5 & 4 & 3 & 5 & 4 & 4 \\
\hline 14 & 2 & 4 & 7 & 2 & 8 & 4 \\
\hline 15 & 5 & 7 & 3 & 7 & 6 & 3 \\
\hline 16 & 6 & 6 & 6 & 3 & 5 & 11 \\
\hline 17 & 3 & 3 & 8 & 6 & 5 & 4 \\
\hline 18 & 2 & 6 & 5 & 6 & 5 & 6 \\
\hline 19 & 5 & 3 & 6 & 4 & 9 & 7 \\
\hline 20 & 3 & 7 & 4 & 6 & 5 & 7 \\
\hline
\end{tabular}

Using a $(1-\mathrm{a})=0.8$, consistent with simulation validation literature (Balci and Sargent 1984, Balci 1994, Kleijnen 1995), confidence intervals were constructed around the simulation means for each scenario assuming a t-distribution with 19 degrees of freedom. The $80 \%$ joint confidence is maintained for each scenario. That is, if $80 \%$ confidence were desired over both scenarios considered together, then the confidence intervals would need to be extended.

Figure 1 shows the results from scenario 1, and the results from scenario 2 are shown in Figure 2. In each case, the confidence intervals either cover or nearly cover the MOE's historical value.

Supposing that the actual number of sightings and kills represent the mean of the true distribution for each scenario, then the joint confidence intervals shown in Figure 1 and Figure 2 would indicate that the simulation does a reasonable job of emulating the scenarios and statistically captures (or nearly captures) the actual values observed during WW II.

Though the results appear to indicate the simulation is a good statistical representation of the historical scenario, two points bear consideration. First, the joint confidence level encompassing both scenarios guarantees a $(1-a)$ significantly less than 0.8 . Second, this conclusion is derived from the assumption that the historical record is representative of the mean of all possible outcomes from the real-world scenario and not a statistical outlier - a decidedly risky assumption. Since there is no way of knowing whether or not this assumption is valid given a "sample 


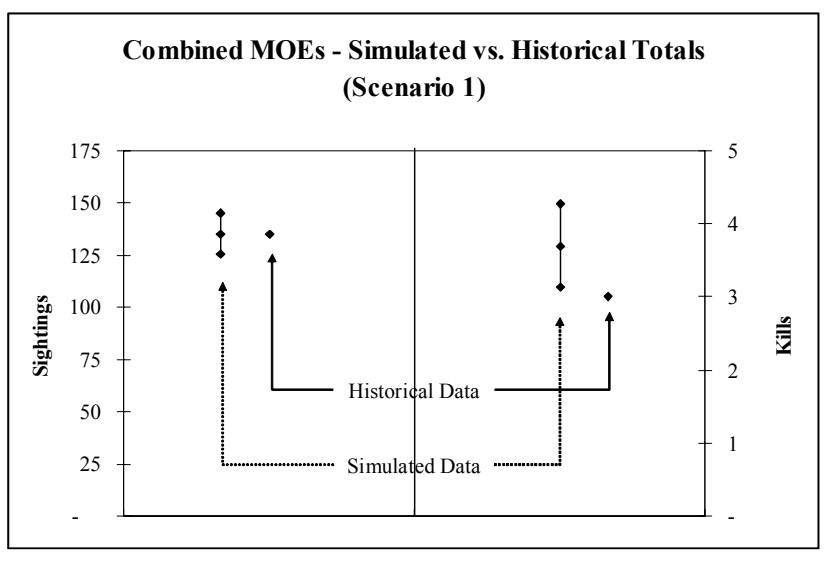

Figure 1: October 1942 - March 1943 MOEs

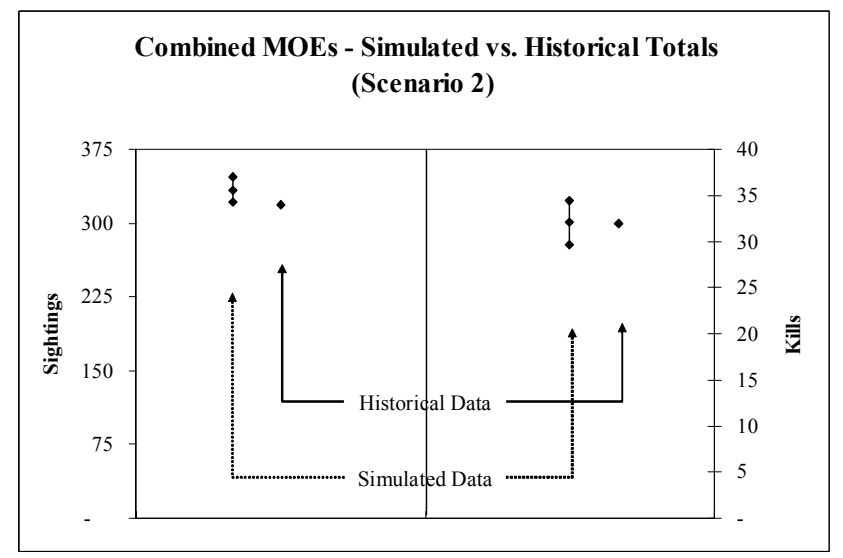

Figure 2: April 1943 - September 1943 MOEs

size of one," conclusions as to the validity of the model should still be considered suspect. However, as a preliminary assessment, the model appears to represent the historical record satisfactorily, and the statistical tests do not contradict this.

\section{MOTIVATION FOR NEW TEST}

While the previous suggests that the simulation is a good representation of the historical scenarios, the fact remains that the historical outcome is itself a single sample from a stochastic process (i.e. combat). The statistical comparisons made in the validation process were based on the assumption that the historic results actually represent the mean value of all possible outcomes. A favorable comparison of the simulation with the underlying stochastic process that produced the single historic sample would provide greater confidence that the model is a valid representation of the real-world system.

Examining Bay of Biscay historic outcomes by month, instead of aggregated, provides a convenient method for examining the variability of the real-world system. Mean monthly values for each MOE of interest, both real-world and simulated, can be calculated and compared. The resulting analysis provides additional insight not available through the previous techniques, although it still lacks quantifiable confidence to conclusions about the validity of the simulation. The data generated from the Bay of Biscay agent-based simulation are used to demonstrate the strengths and weaknesses of this approach.

Figures 3 through 6 depict the historic versus simulated mean monthly MOE values via joint confidence intervals each MOE, U-Boat sightings and kills, in both scenarios, respectively. Each figure shows 21 individual confidence intervals - the left-most being the historic value with the remaining 20 coming from each of 20 simulation iterations. Joint confidence intervals were constructed to allow an overall $80 \%$ joint confidence level $(\mathrm{k}=$ 2) for each comparison.

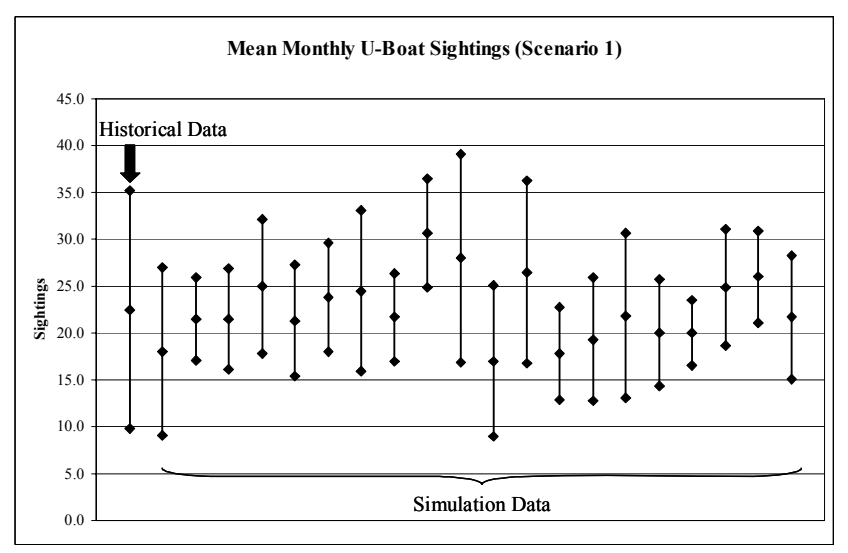

Figure 3: Comparisons of Mean Monthly U-Boat Sightings, Historic vs. Simulated Scenario 1

Even with no further analysis, a major shortcoming of this validation approach becomes evident. In preparing for the comparisons, an analyst must choose two unattractive options when constructing joint confidence intervals. The first option is to compare each simulation iteration to the historic data at some known confidence level (e.g. 80\% with $\mathrm{k}=2$, as presented in Figures 3 through 6). The second option is to construct the intervals such that all simulation iterations versus historic outcome comparisons taken together have a known joint confidence level (i.e. $\mathrm{k}=21$ ). If the former option is chosen, the resulting joint confidence level for all 20 comparisons is near zero. If the latter is chosen, the overall confidence level is known, but the individual confidence intervals are so large they cease to be discriminating. 


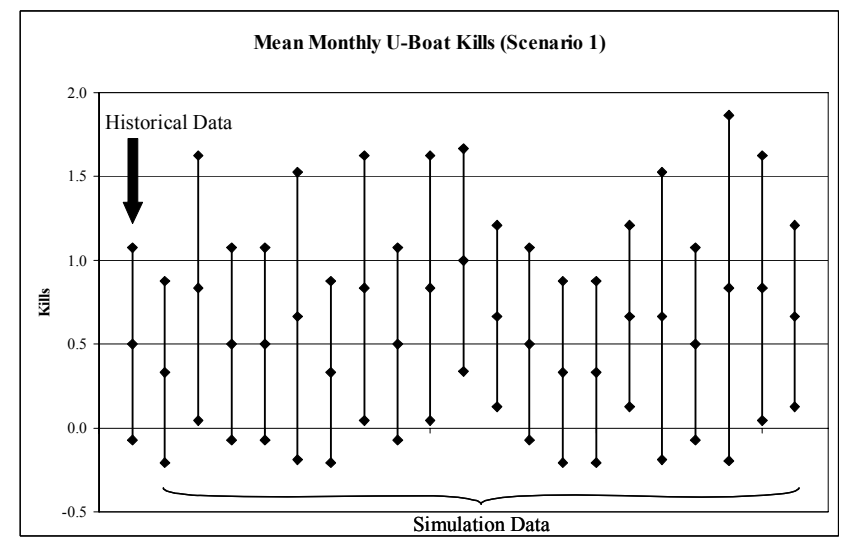

Figure 4: Comparisons of Mean Monthly U-Boat Kills, Historic vs. Simulated Scenario 1

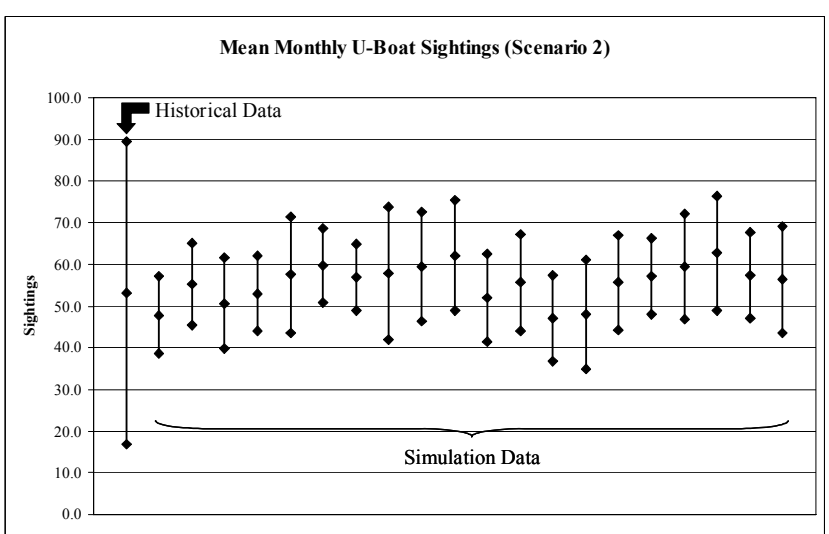

Figure 5: Comparisons of Mean Monthly U-Boat Sightings, Historic vs. Simulated Scenario 2

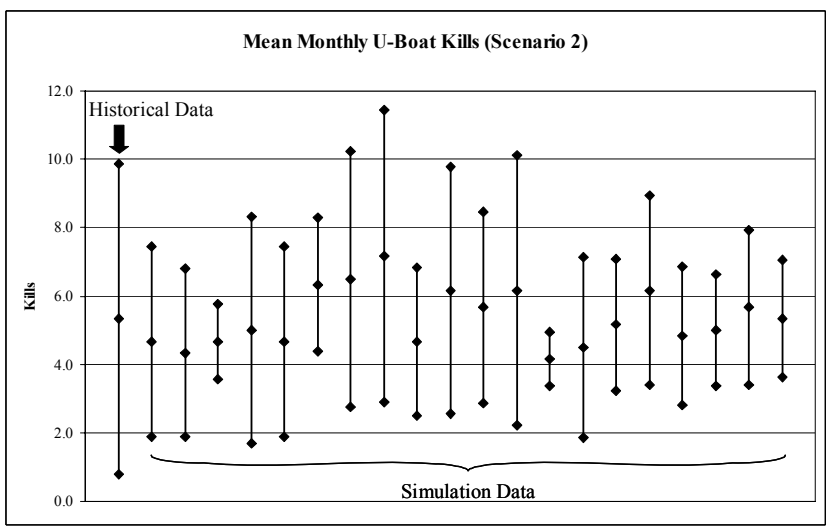

Figure 6: Comparisons of Mean Monthly U-Boat Kills, Historic vs. Simulated Scenario 2

Figures 3-6 indicate 100\% confidence level overlap in comparing individual simulation iteration means to the real-world data for each MOE. These figures provide facelevel support for this conclusion.
However, because of the analytic dilemma surrounding the joint confidence level, this method of analysis provides little more than face-level confidence. The statistical confidence remains near zero. However, the approach is tempting in that it offers insight into the stochastic nature underlying a real-world system with a single occurrence (sample size of 1). we next present and demonstrate a methodology that allows for statistically significant comparisons, despite having a single real-world sample.

\subsection{Methodology For Comparison Of Historic Versus Simulated Data}

Any test allowing a meaningful comparison between the historic outcome and the simulated data, while still providing insight into the underlying stochastic real-world system, requires two characteristics. First, the method must provide a means of deriving multiple samples from the stochastic process underlying the real-world system. Second, the method must provide a meaningful, quantifiable level of confidence in the result. Figure 7 illustrates an approach that meets both requirements.

Once the simulation results from $n$ iterations are generated, the historic data is used to generate $n$ bootstrap samples. A sign test is used to test the hypothesis that the two samples are statistically identical. The bootstrap and sign test is then replicated for multiple experiments.

The basic approach above is based on well-accepted nonparametric statistical techniques. Once the simulation data has been collected, the approach has the added benefit of being simple to execute and can be quickly performed within a spreadsheet.

\subsubsection{Bootstrap}

Several statistical resampling techniques have been developed to provide estimators of population parameters that are difficult or impossible to treat theoretically (Conover 1999) or when obtaining multiple samples from a system is prohibitively expensive (Cheng 2001). Resampling is based on the idea that when one random sample is available and obtaining another sample is not feasible, then the best estimate for the distribution under study is the random sample in-hand.

Efron (1979) first proposed the bootstrap method of resampling. Since it was first proposed, the method has found wide acceptance and applicability. Efron and Tibshirani (1986) review the bootstrap method and its applications. 


\section{Champagne and Hill}

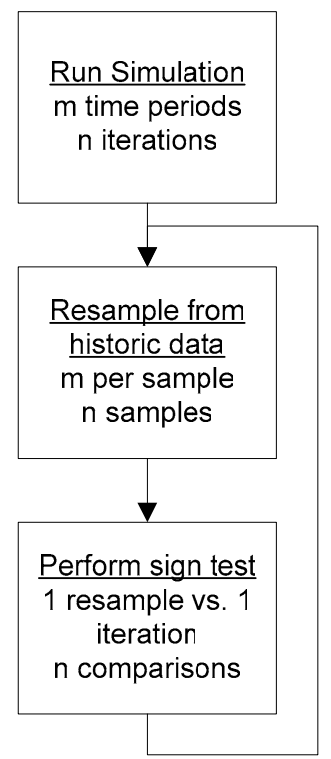

Figure 7: Methodology for Comparisons of a SingleSampled Real-World Process to Simulated Results

The Method. Consider the statistic q calculated from the random sample $\mathrm{X}=\left\{\mathrm{X}_{1}, \mathrm{X}_{2}, \ldots, \mathrm{X}_{\mathrm{n}}\right\}$. A bootstrap sample $\mathrm{X}^{*}=\left\{X_{1}^{*}, X_{2}^{*}, \ldots, X_{n}^{*}\right\}$ is generated by taking a random sample from $X$, where $P\left(X_{j}^{*}(j=1,2, \ldots, n)=X_{i}(i=1,2, \ldots, n)\right)=\frac{1}{n}$, for which $\mathrm{q}^{*}$, an estimator for $\mathrm{q}$, is computed from the bootstrap sample. If some number, $B$, Monte Carlo replications are taken, then the distribution of q can be estimated by the sample mean and standard deviation of $\mathrm{q}^{*}$.

Sample Size, B. The number of bootstrap samples needed to accurately estimate the properties of the sample statistic vary. Efron and Tibshirani (1986) note that for most situations, $\mathrm{B}=50$ to 200 is "quite adequate," though 250 or more are often needed for accurate computation of confidence intervals. Conover (1999) adds that "as few as 25 replications can be very informative".

Proposed Use. The bootstrap used differs slightly for the proposed methodology. Instead of a single collection of bootstrap samples of the historic data, $m$ groups of $b$ bootstrap samples were generated for comparison with the simulation, where $b=$ the number of simulation iterations and $\mathrm{m}=$ number of sign test trials desired.

Assumptions and Remedial Methods. Bootstrap resampling assumes the original sample is independent and identically distributed (i.i.d.). Since the historic data from the Bay of Biscay operations consists of calendar data (i.e. time-series data), it is likely that the MOE data is autocor- related to some degree. Table 7 shows the calculated autocorrelation (1 time lag) for the data from each Scenario.

Table 7: Autocorrelation of Historic MOE Values

\begin{tabular}{|l|r|r|}
\cline { 2 - 3 } \multicolumn{1}{c|}{} & Scenario 1 & Scenario 2 \\
\hline U-Boat Sightings & 0.5345 & 0.1192 \\
\hline U-Boat Kills & 0.1667 & -0.3189 \\
\hline
\end{tabular}

From Table 7, it appears that autocorrelation is an issue with Scenario 1 U-Boat Sightings and Scenario 2 U-Boat kills. Statistically, however, the extremely small sample size $(n=6)$ for both Scenarios does not provide any conclusive evidence that the samples are autocorrelated. This small sample size also prevents the practical application of remedial data measures that could treat the correlation within the samples. There are methods of treating autocorrelated samples so that the bootstrap assumptions can be met. The moving blocks bootstrap is one method that extends the bootstrap to time series data (Dixon, 2001).

In the moving blocks bootstrap, the time series data is partitioned into $b$ non-overlapping blocks consisting of $l$ sequential observations. Values of $b$ and $l$ are chosen so that the correlation within each of the blocks is strong, but weak between blocks. With $l$ correctly chosen, the $b$ blocks are considered independent. The bootstrap method randomly samples with replacement from the $b$ blocks to obtain a series of $b \cdot l$ observations.

The moving blocks bootstrap is not a feasible solution to the specific problem posed by the Bay of Biscay scenario validation data. The small number of observations in each validation set prevents effective blocking schemes. The fidelity of the available data also represents an obstacle. Data for the Bay of Biscay operations are available in monthly increments (observations). If the data were available in smaller time increments (more observations), then perhaps a viable blocking scheme could be contrived.

Combat operations will perpetually pose sample size problems since real-world operations seldom maintain stationary/static strategies, tactics, or technologies long enough to produce data of a significant sample size.

\subsubsection{Sign Test}

The sign test is used to test whether one random variable in a pair $(\mathrm{X}, \mathrm{Y})$ tends to be larger than the other random variable in the pair. It is a variant of the binomial test in which the probability of outcome is assumed to be equally likely, $\mathrm{p}=1-\mathrm{p}=0.5$ (Conover 1999).

Data for the sign test consists of $n$ ' pairs of observations $\left(X_{1}, Y_{1}\right),\left(X_{2}, Y_{2}\right), \ldots,\left(X_{n}, Y_{n}\right)$, each observation being a bivariate random sample. Within each $\left(\mathrm{X}_{\mathrm{i}}, \mathrm{Y}_{\mathrm{i}}\right)$ observation, a comparison is made, and the pair is classified as " + " if $X_{i}<Y_{i}$, " - " if $X_{i}>Y_{i}$, or " 0 " if $X_{i}=Y_{i}$. The test statistic, $T$, is the number of "+" pairs. The null distribu- 


\section{Champagne and Hill}

tion of $T$ is the binomial distribution with $p=1 / 2$ and $n=$ number of non-tied pairs (tied pairs are disregarded).

The sign test assumes that the bivariate pairs are mutually independent, and the probability of outcome is constant for all trials. It further assumes that the measurement scale within each pair is at least ordinal, that is each $\left(\mathrm{X}_{\mathrm{i}}\right.$, $\mathrm{Y}_{\mathrm{i}}$ ) pair may be determined to be “+”, “-”, or " 0 ". Finally, the sign test assumes there is internal consistency between the observed pairs.

For model validation purposes, the two-tailed test is desired. That is,

$$
\begin{gathered}
\mathrm{H}_{0}: \mathrm{P}(+)=\mathrm{P}(-) \\
\mathrm{H}_{1}: \mathrm{P}(+) \neq \mathrm{P}(-) .
\end{gathered}
$$

The critical a-values are determined for each test once $n$ has been determined. Because the binomial distribution is discrete, the critical a-values cannot be arbitrarily set. Instead, the critical a-level is selected such that the total (1 - a) level is as close to 0.9 as possible, without being less than 0.9 , given a particular $n$. That is, $\mathrm{H}_{0}$ is rejected if the $\mathrm{p}$-value for the test is less than 0.05 .

\subsection{Bay Of Biscay Agent-Based Simulation Results}

The presentation of results follows the same order as in the previous analyses. That is, the comparisons of sortie hours for both scenarios are presented first, followed by the remaining MOEs from each scenario, respectively.

Each MOE was subjected to identical experiments. Each experiment consists of twenty sign tests $(m=20)$, with each sign test incorporating twenty (one per simulation iteration) bootstrap samples $(b=20)$. For each MOE, one sign test is presented in detail, and the remaining tests are summarized prior to validation discussions.

\subsubsection{Scenario 1 MOEs}

Previous analyses of Scenario 1 MOEs provided a somewhat mixed picture of the simulation's fidelity with respect to the historic data. The historic U-Boat kills total was slightly outside the simulation confidence interval, though the practical difference was negligible. Comparisons between the confidence interval generated by the historic monthly data and those generated from each iteration's monthly data, however, demonstrated $100 \%$ overlap, and hence, no statistical difference between the results from any individual iteration and the historic data. This approach also lacked any meaningful confidence when all such comparisons were taken together. The historic UBoat sightings total was well within the confidence interval derived from the simulation data. The subsequent analysis with respect to the monthly means showed similar results to the U-Boat kills with the identical problem of providing no joint confidence.
Table 8 shows the bootstrap samples for Scenario 1 UBoat sightings generated for comparison with the simulation results.

Table 9 summarizes the sign test classifications for the paired data $\left(\mathrm{X}_{\mathrm{i}}, \mathrm{Y}_{\mathrm{i}}\right)$ for Scenario $1 \mathrm{U}$-Boat sightings, where $\mathrm{X}_{\mathrm{i}}$ is the $i^{\text {th }}$ bootstrap U-Boat sightings total and $\mathrm{Y}_{\mathrm{i}}$ is the U-Boat sightings total from the $i^{\text {th }}$ simulation iteration. The sign test statistic $T$ and number of non-tied pairs $n$ are displayed as well.

Table 8: Bootstrap U-Boat Sightings - Scenario 1

\begin{tabular}{|r|r|r|r|r|r|r|}
\hline Trial & $10 / 42$ & $11 / 42$ & $12 / 42$ & $1 / 43$ & \multicolumn{1}{l}{$2 / 43$} & $3 / 43$ \\
\hline 1 & 14 & 18 & 10 & 42 & 42 & 42 \\
\hline 2 & 18 & 14 & 42 & 18 & 19 & 18 \\
\hline 3 & 18 & 18 & 19 & 18 & 19 & 14 \\
\hline 4 & 10 & 14 & 14 & 14 & 42 & 14 \\
\hline 5 & 14 & 19 & 42 & 32 & 42 & 19 \\
\hline 6 & 42 & 18 & 32 & 32 & 42 & 14 \\
\hline 7 & 19 & 32 & 14 & 32 & 18 & 19 \\
\hline 8 & 18 & 14 & 14 & 10 & 14 & 42 \\
\hline 9 & 18 & 19 & 18 & 42 & 18 & 19 \\
\hline 10 & 32 & 32 & 32 & 32 & 18 & 18 \\
\hline 11 & 32 & 10 & 19 & 14 & 10 & 32 \\
\hline 12 & 10 & 19 & 42 & 32 & 10 & 32 \\
\hline 13 & 32 & 19 & 19 & 42 & 18 & 18 \\
\hline 14 & 32 & 32 & 42 & 42 & 42 & 10 \\
\hline 15 & 10 & 32 & 14 & 18 & 18 & 32 \\
\hline 16 & 32 & 32 & 10 & 18 & 42 & 14 \\
\hline 17 & 19 & 19 & 14 & 19 & 19 & 32 \\
\hline 18 & 32 & 19 & 42 & 18 & 32 & 14 \\
\hline 19 & 10 & 19 & 19 & 32 & 32 & 32 \\
\hline 20 & 32 & 42 & 10 & 32 & 42 & 14 \\
\hline
\end{tabular}

Table 9: Sign Test Calculations - U-Boat Sightings, Scenario 1

\begin{tabular}{|l|c|c|c|c|c|c|c|c|c|c|}
\hline $\begin{array}{l}\text { Ob- } \\
\text { ser- } \\
\text { va- } \\
\text { tion }\end{array}$ & 1 & 2 & 3 & 4 & 5 & 6 & 7 & 8 & 9 & 10 \\
\hline Sign & - & 0 & + & + & - & - & + & + & + & + \\
\hline \hline $\begin{array}{l}\text { Ob- } \\
\text { ser- } \\
\text { va- } \\
\text { tion }\end{array}$ & 11 & 12 & 13 & 14 & 15 & 16 & 17 & 18 & 19 & 20 \\
\hline Sign & - & + & - & - & + & - & - & - & + & - \\
\hline \hline $\mathrm{T}$ & 9 & \multicolumn{10}{|l}{} & & & & & & & & \\
\hline $\mathrm{n}$ & 19 &
\end{tabular}

For $\mathrm{n}=19, \mathrm{P}(t \leq 5)=0.0358$ and $\mathrm{P}(t \geq 13)=0.0358$ defining an overall $(1-\mathrm{a})=0.9284$. Since $5<T=9<13$, there is insufficient evidence to reject $\mathrm{H}_{0}$. There is no compelling evidence to suggest the simulation does not 
faithfully represent the real-world system with respect to Scenario 1 U-Boat sightings.

Table 10 shows the bootstrap samples of Scenario 1 U-Boat kills generated for a single replication of the bootstrap/sign test experiment. Of the 20 sign test trials, the pvalues ranged in value from 0.011 to 0.5 . Under the rejection criteria, the null hypothesis was rejected in 5 of the 20 trials.

Both sign test experiments tend to indicate that the simulation is representative of historical combat operations for Scenario 1. In the case of Scenario $1 \mathrm{U}$-Boat sightings, the bootstrap/sign test rejected the null hypothesis in $15 \%$ of the trials. With respect to Scenario 1 U-Boat kills, the bootstrap/sign test method rejected the null hypothesis in $25 \%$ of the trials. Rather than make a validation conclusion based on a single statistical pass/fail, as in the first analysis method, the bootstrap/sign test methodology provides a broader context to the simulation results. These conclusions provide stronger rationale than either of the previous tests for accepting the model as valid with respect to the MOEs.

\subsubsection{Scenario 2 MOEs}

Previous analyses of Scenario 2 MOEs provided a somewhat mixed picture of the simulation's fidelity with respect to the historic data. The historic U-Boat sightings total was slightly outside the simulation confidence interval, though the practical difference was negligible. Comparisons between the confidence interval generated by the historic monthly data and those generated from each iteration's monthly data, however, demonstrated $100 \%$ overlap, and hence, no statistical difference between the results from any individual iteration and the historic data. This approach, however, also lacked any meaningful confidence when all such comparisons were taken together. The historic U-Boat kills total was well within the confidence interval derived from the simulation data. The subsequent analysis with respect to the monthly means showed similar results to the sightings with the identical joint confidence problem.

Table 11 shows the bootstrap samples for Scenario 2 U-Boat sightings generated for a single replication of the bootstrap/sign test experiment.

Of the 20 sign test trials, the p-values ranged in value from 0.058 to 0.412 . Under the rejection criteria, the null hypothesis was not rejected in any of the 20 trials.

Table 12 shows the bootstrap samples of Scenario 2 U-Boat kills. Of the 20 sign test trials, the p-values ranged in value from 0.058 to 0.5 . Under the rejection criteria, the null hypothesis was not rejected in any of the 20 trials.
Table 10: Bootstrap U-Boat Kills - Scenario 1

\begin{tabular}{|l|r|r|r|r|r|r|}
\hline Trial & $10 / 42$ & $11 / 42$ & $12 / 42$ & $1 / 43$ & \multicolumn{1}{l|}{$2 / 43$} & \multicolumn{1}{l|}{$3 / 43$} \\
\hline 1 & 0 & 0 & 1 & 0 & 1 & 1 \\
\hline 2 & 1 & 1 & 1 & 1 & 0 & 1 \\
\hline 3 & 1 & 0 & 0 & 1 & 0 & 0 \\
\hline 4 & 1 & 0 & 1 & 1 & 0 & 1 \\
\hline 5 & 0 & 1 & 1 & 1 & 0 & 0 \\
\hline 6 & 0 & 0 & 1 & 1 & 0 & 1 \\
\hline 7 & 0 & 1 & 1 & 1 & 0 & 1 \\
\hline 8 & 0 & 1 & 1 & 0 & 1 & 1 \\
\hline 9 & 1 & 1 & 1 & 1 & 1 & 1 \\
\hline 10 & 0 & 1 & 0 & 1 & 0 & 0 \\
\hline 11 & 0 & 0 & 1 & 1 & 1 & 1 \\
\hline 12 & 1 & 0 & 1 & 1 & 1 & 1 \\
\hline 13 & 0 & 0 & 0 & 1 & 1 & 1 \\
\hline 14 & 0 & 1 & 0 & 1 & 1 & 1 \\
\hline 15 & 1 & 0 & 1 & 1 & 0 & 0 \\
\hline 16 & 0 & 0 & 1 & 0 & 0 & 1 \\
\hline 17 & 1 & 1 & 0 & 1 & 1 & 1 \\
\hline 18 & 0 & 1 & 1 & 1 & 0 & 0 \\
\hline 19 & 1 & 0 & 1 & 0 & 0 & 1 \\
\hline 20 & 0 & 0 & 1 & 1 & 0 & 1 \\
\hline
\end{tabular}

Table 11: Bootstrap U-Boat Sightings - Scenario 2

\begin{tabular}{|l|r|r|r|r|r|r|}
\hline Trial & \multicolumn{1}{|c|}{$4 / 43$} & \multicolumn{1}{|c|}{$5 / 43$} & $6 / 43$ & $7 / 43$ & $8 / 43$ & \multicolumn{1}{|c|}{$9 / 43$} \\
\hline 1 & 81 & 7 & 52 & 60 & 98 & 52 \\
\hline 2 & 98 & 98 & 21 & 98 & 81 & 98 \\
\hline 3 & 98 & 81 & 81 & 21 & 60 & 7 \\
\hline 4 & 98 & 7 & 52 & 52 & 60 & 52 \\
\hline 5 & 81 & 52 & 52 & 52 & 60 & 60 \\
\hline 6 & 81 & 81 & 98 & 52 & 7 & 52 \\
\hline 7 & 60 & 98 & 98 & 21 & 7 & 21 \\
\hline 8 & 7 & 52 & 98 & 81 & 21 & 98 \\
\hline 9 & 52 & 52 & 52 & 52 & 21 & 98 \\
\hline 10 & 60 & 98 & 60 & 52 & 81 & 60 \\
\hline 11 & 81 & 81 & 21 & 21 & 52 & 98 \\
\hline 12 & 98 & 60 & 21 & 52 & 52 & 21 \\
\hline 13 & 60 & 7 & 81 & 52 & 21 & 52 \\
\hline 14 & 7 & 52 & 60 & 52 & 21 & 52 \\
\hline 15 & 52 & 81 & 98 & 21 & 81 & 81 \\
\hline 16 & 7 & 81 & 21 & 60 & 81 & 52 \\
\hline 17 & 98 & 52 & 7 & 21 & 21 & 21 \\
\hline 18 & 60 & 98 & 98 & 21 & 7 & 60 \\
\hline 19 & 52 & 60 & 21 & 81 & 81 & 98 \\
\hline 20 & 7 & 81 & 98 & 21 & 81 & 21 \\
\hline
\end{tabular}

Both sign test experiments indicate the simulation is representative of historical combat operations for Scenario 2; null hypothesis was not rejected in 20 trials for either MOE. Though the original validation test showed a statistical difference in the number of U-Boat sightings, the results of the sign test indicate the simulation was a better 


\section{Champagne and Hill}

model than the original test indicated. The monthly mean test demonstrated $100 \%$ overlap between the historic and simulation confidence intervals. The conclusions drawn from the bootstrap/sign test methodology provide stronger indication than either of the previous tests for accepting the model as valid with respect to the MOEs.

Table 12: Bootstrap U-Boat Kills - Scenario 2

\begin{tabular}{|r|r|r|r|r|r|r|}
\hline Trial & \multicolumn{1}{|l|}{$4 / 43$} & \multicolumn{1}{|l}{$5 / 43$} & \multicolumn{1}{l|}{$6 / 43$} & \multicolumn{1}{l|}{$7 / 43$} & $8 / 43$ & $9 / 43$ \\
\hline 1 & 4 & 4 & 1 & 2 & 1 & 13 \\
\hline 2 & 4 & 13 & 1 & 13 & 5 & 2 \\
\hline 3 & 4 & 4 & 1 & 5 & 7 & 2 \\
\hline 4 & 1 & 2 & 7 & 5 & 2 & 13 \\
\hline 5 & 2 & 7 & 1 & 1 & 4 & 1 \\
\hline 6 & 7 & 1 & 5 & 1 & 2 & 5 \\
\hline 7 & 2 & 4 & 1 & 5 & 1 & 13 \\
\hline 8 & 1 & 5 & 1 & 5 & 7 & 4 \\
\hline 9 & 13 & 5 & 5 & 7 & 5 & 7 \\
\hline 10 & 13 & 13 & 5 & 1 & 5 & 5 \\
\hline 11 & 4 & 1 & 1 & 2 & 1 & 2 \\
\hline 12 & 1 & 7 & 1 & 1 & 1 & 2 \\
\hline 13 & 13 & 5 & 13 & 1 & 2 & 1 \\
\hline 14 & 13 & 4 & 2 & 5 & 2 & 1 \\
\hline 15 & 2 & 7 & 13 & 4 & 13 & 13 \\
\hline 16 & 4 & 1 & 5 & 13 & 13 & 1 \\
\hline 17 & 13 & 2 & 13 & 13 & 1 & 1 \\
\hline 18 & 4 & 7 & 13 & 5 & 1 & 7 \\
\hline 19 & 4 & 4 & 5 & 7 & 2 & 7 \\
\hline 20 & 5 & 7 & 7 & 7 & 7 & 13 \\
\hline
\end{tabular}

\section{VALIDATION CONCLUSIONS}

In the first validation analysis, the traditional t-test showed half of the six tests with statistical difference between the simulation and historic data, although the practical differences were essentially negligible. These tests assumed the historic outcome represented the mean of all such outcomes - a possibly risky assumption.

In the second validation analysis, the simulation appeared to perform exceedingly well against the real-world data. However, due to the joint confidence dilemma discussed previously, little insight could be made with practical statistical confidence.

The proposed bootstrap/sign test validation methodology provides more information either traditional method. The sortie hour tests produced null hypothesis rejection rate of $15 \%$ for Scenario 1 and $5 \%$ for Scenario 2 . The remaining MOEs for Scenario 1 produced a null hypothesis rejection rate of $15 \%$ for U-Boat sightings and $25 \%$ for UBoat kills. Scenario 2 produced a null hypothesis rejection rate of $0 \%$ for both MOEs.

Ultimately, the validation determination rests with the decision maker, who takes risk, practical differences, and other associated costs into account. Our experiences and test suggest the BoB model is sufficiently valid, and its success as an experimental platform has been demonstrated and well documented in (Champagne, Carl, and Hill 2003a), (Champagne, Carl, and Hill 2003b), (Champagne and Hill 2003), (Carl 2003), and (Hill, Price, and Champagne 2003).

\section{ACKNOWLEDGMENTS}

This research was funded by the Defense Modeling and Simulation Office (DMSO) and the Air Force Research Laboratory, Human Effectiveness Directorate (AFRL/HES).

\section{REFERENCES}

Balci, O. and R. G. Sargent. 1984. Validation of simulation models via simultaneous confidence intervals. American Journal of Mathematical and Management Sciences 4: 375-406.

Balci, O. 1994. Validation, verification, and testing throughout the life cycle of a simulation study. Annals of Operation Research 54: 121-174.

Brown, L. 2000. Agent based simulation as an exploratory tool in the study of the human dimension of combat. Monterey, CA: Naval Postgraduate School.

Bullock, R. K. 2000. Hierarchical Interactive Theater Model (HITM). WPAFB, OH: Air Force Institute of Technology. AFIT/GOA/ENS/00M-05.

Carl, R. G. 2003. Search theory and U-boats in the Bay of Biscay. OR/MS Tomorrow. Spring 2003.

Champagne, L. 2003a. Development approaches coupled with verification and validation methodologies for agent-based mission-level analytical combat simulations. WPAFB, OH: Air Force Institute of Technology. AFIT/DS/ENS/03-02.

Champagne, L. 2003b. Bay of Biscay: Extensions into modern military issues. Proceedings of the 2003 Winter Simulation Conference, ed. S. Chick, P.J. Sanchez, D. Ferrin, and D. J. Morrice, 1004-1012. Piscataway, New Jersey: Institute of Electrical and Electronics Engineers, New Orleans, LA.

Champagne, L. and R. Hill. 2003. Multi-agent simulation analysis: Bay of Biscay case study. Proceedings of SimTecT 2003. Adelaide, Australia. May 26-29.

Champagne, L., R. G. Carl, and R. Hill. 2003a. Multi-agent techniques: Hunting U-boats in the Bay of Biscay. Proceedings of SimTecT 2003. Adelaide, Australia. May 26-29.

Champagne, L., R. G. Carl, and R. Hill. 2003b. Search theory, agent-based simulation, and u-boats in the bay of Biscay. Proceedings of the 2003 Winter Simulation Conference, ed. S. Chick, P.J. Sanchez, D. Ferrin, and D. J. Morrice, 991-998. Piscataway, New Jersey: Institute of Electrical and Electronics Engineers. New Orleans, LA. 


\section{Champagne and Hill}

Cheng, R. 2001. Analysis of simulation experiments by bootstrap resampling. Proceedings of the 2001 Winter Simulation Conference. B. A. Peters, J. S. Smith, D. J. Medeiros, and M. W. Rohrer, 179-186. Piscataway, New Jersey: Institute of Electrical and Electronics Engineers.

Conover, W. J. 1999. Practical nonparametric statistics, third edition. John Wiley and Sons, Inc.: New York, New York.

Dixon, P. M. 2001. The bootstrap. Department of Statistics, Iowa State University.

Efron, B. 1979. Bootstrap methods: another look at the jacknife. Annals of Statistics 7: 1-26.

Efron, B. and R. Tibshirani. 1986. Bootstrap methods for standard errors, confidence intervals and other measures of statistical accuracy. Statistical Science 1: 5477.

Erlenbruch, T. 2002. Agent-based simulation of German peacekeeping operations for units up to platoon level. Monterey, CA: Naval Postgraduate School.

Hill, R., J. Price, and L. Champagne. 2003. Agent Modeling with Game Theory Constructs. Summer Simulation Conference, 2003. Montreal, Canada. July.

Ilachinski, A. 1998. Irreducible semi-autonomous adaptive combat (ISAAC). Maneuver Warfare Science 1998. ed. F.G. Hoffman and Gary Horne. United States Marine Corps.

Kleijnen, J. P. C. 1995. Statistical validation of simulation models. European Journal of Operational Research 87: 21-34.

Levy, S. 1992. Artificial life: A report from the frontier where computers meet biology. New York: Vintage Books, a division of Random House, Inc.

McCue, B. 1990. U-boats in the Bay of Biscay: An essay in operations research. Washington DC: National Defense University Press.

Morse, P. M. and G. E. Kimball. 1998. Methods of Operations Research. Alexandria, Virginia: Military Operation Research Society. Reprinted in its entirety from (C) 1951 first edition printed by MIT Press and John Wiley \& Sons, Inc.

Tighe, T. 1999. Strategic effects of airpower and complex adaptive agents: an initial investigation. WPAFB, OH: Air Force Institute of Technology. AFIT/GOA/ENS/99M-09.

Waddington, C. H. 1973. O.R. in World War 2: Operational research against the U-boat. London, England: Paul Elek (Scientific Books) Ltd.

Woodaman, R. 2000. Agent-based simulation of military operations other than war small unit. Monterey, CA: Naval Postgraduate School.

\section{AUTHOR BIOGRAPHIES}

LANCE E. CHAMPAGNE is a Major in the United States Air Force and Chief Analyst for the Air Force Logistics Management Agency. He has a Ph.D. in Operations Research from the Air Force Institute of Technology. His research interests include agent-based modeling and verification and validation methodology. His email address is lance. champagne@maxwel1.af .mil.

RAYMOND R. HILL is an Associate Professor of Industrial and Human Factors Engineering with Wright State University. He has a Ph.D. from the Ohio State University and has research interests in heuristic analysis, applied optimization and simulation modeling. His email address is ray.hill@wright. edu.

Distribution: DISTRIBUTION A. Approved for public release; distribution unlimited.

Disclaimer: The views expressed in this article are those of the authors and do not reflect the official policy of the United States Air Force, Department of Defense, or the US Government. 